\title{
Upgrade of the ATLAS Level-1 Calorimeter Trigger
}

\author{
Felix Müller on behalf of the ATLAS collaboration
}

\begin{abstract}
The Level-1 calorimeter trigger (L1Calo) operated successfully during the first data taking phase of the ATLAS experiment at the LHC. Facing the new challenges posed by the upcoming increases of the LHC beam energy and luminosity, and from the experience of the previous running, a series of upgrades is planned for L1Calo. The initial upgrade phase in 2013-14 includes substantial improvements to the analogue and digital signal processing to cope with baseline shifts due to signal pile-up. Additionally a newly introduced system will receive realtime data from both the upgraded L1Calo and L1Muon trigger to perform trigger algorithms based on entire event topologies. During the second upgrade phase in 2018-19 major parts of L1Calo will be rebuilt in order to exploit a tenfold increase in the available calorimeter data granularity compared to that of the current system.

This contribution gives an overview of the existing system and the lessons learned during the first period of LHC data taking. Based on these, the expected performance improvements and the hardware implementations for the initial upgrade are discussed. Furthermore, the plans for the second upgrade phase are outlined.
\end{abstract}

Index Terms-ATLAS, trigger, upgrade.

\section{INTRODUCTION}

$\mathbf{T}$ HE ATLAS Level-1 Calorimeter Trigger (L1Calo) was operated very successfully during the initial data taking phase of the ATLAS experiment [1]. It played a major role in the discovery of the Higgs boson. Beyond this very important step, the LHC holds a longterm programme as a discovery machine. In the upcoming data taking period, the LHC will increase the centre-of-mass energy by a factor of two. At the same time, it aims to collect an extremely large dataset for precision measurements, e.g. of the properties of the Higgs boson.

The schedule of the LHC is as follows. In the years 2009 to 2011 , the LHC was operated at $7 \mathrm{TeV}$ and $8 \mathrm{TeV}$ centre-ofmass energy. The integrated luminosity of the ATLAS Run 1 dataset amounts to $25 \mathrm{fb}^{-1}$, while the instantaneous luminosity reached up to $70 \%$ of its nominal value of $L=10^{34} \mathrm{~cm}^{-2} \mathrm{~s}^{-1}$. Currently, the LHC is shutdown for an upgrade to reach the designed centre-of-mass energy of $14 \mathrm{TeV}$. This initial upgrade programme is called Phase 0 throughout this contribution. In 2015, the LHC will run with the nominal instantaneous luminosity of $L=3 \times 10^{34} \mathrm{~cm}^{-2} \mathrm{~s}^{-1}$ and a centre-of-mass energy of $13-14 \mathrm{TeV}$. The data taking period of Run 2 is foreseen to end in 2018. In the high luminosity upgrade of the LHC, an increase of the instantaneous luminosity to about twice the design luminosity of the initial concept is planned. This second large upgrade before the beginning of Run 3 in 2019 is called Phase 1.

The Phase 0 upgrade of the LHC poses new demands on the ATLAS trigger system. Although the Level-1 rate will be increased from about $70 \mathrm{kHz}$ during Run 1 to $100 \mathrm{kHz}$ for Run 2,

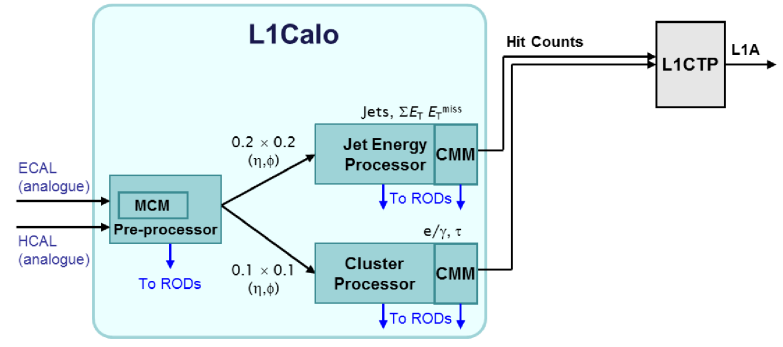

Fig. 1. Block diagram of the Level-1 Calorimeter Trigger system during Run 1 [4].

the increase of the collision energy from $8 \mathrm{TeV}$ to $14 \mathrm{TeV}$ as well as the anticipated rise of the instantaneous luminosity in Run 2 require a tightening of the trigger requirements. In particular, an improved suppression of effects due to multiple collisions in the same bunch crossing (in-time pile-up) or from residual signals in preceeding bunch crossings (out-of-time pile-up) is crucial. Furthermore, new approaches to select the events of interest from the background are required, such as exploiting the topology of the event.

The high luminosity upgrade of the LHC in Phase 1 will lead to even higher particle occupancies and requires a better selectivity for the trigger. Therefore, a tenfold increase of the granularity with respect to the current system is planned for the Phase 1 upgrade of L1Calo, e.g. allowing to make use of the shower shape. This includes a substantial change in the read-out of the calorimeter, performing the digitisation of the trigger signals on-detector and changing to optical data transmission for the calorimeter based on liquid Argon (LAr) technology. Accordingly, a major rebuild of the Level-1 Calorimeter Trigger processors is undertaken.

\section{SyStem ARCHITECTURE}

The real-time data path of the Level-1 Calorimeter Trigger consists of three major building blocks: the PreProcessor (PPr), the Cluster Processor (CP), the Jet/Energy Processor (JEP), which are complemented by the Common Merger Modules (CMM). In addition, the system includes the Read-Out Drivers (RODs) for data acquisition and monitoring of the trigger system. A block diagram of the system is shown in Figure 1.

The input are 7198 analogue signals from the calorimeter, so-called trigger towers. In the region $|\eta|<2.5$, each signal is the sum of calorimeter cells in an area of $0.1 \times 0.1$ in $\eta-\phi$ space, with a longitudinal separation into an electromagnetic and a hadronic layer. In the forward region, the size of the trigger towers increases to up to $0.4 \times 0.4$. The main functionality of the PPr is hosted on a mezzanine card, the socalled Multi-Chip Module (MCM). It performs the digitisation 
of the analogue signals at a frequency of $40 \mathrm{MHz}$, using a 10bit flash ADC. The pulse sampling can be shifted in steps of $1 \mathrm{~ns}$ to allow for a precise sampling of the signal peak. The PPr provides the alignment of the input signals to a common time frame, accounting for the different time-of-flight of the particles in the detector and different signal propagation times in the cables. The digitised signals are processed by a finite impulse response filter and a subsequent peak finder, where the identification of the corresponding bunch crossing takes place. The conversion of ADC counts to the corresponding transverse energy at electromagnetic scale is done using a look-up table. The output is sent in parallel to the two subsequent digital processors, CP and JEP, using a LVDS serialiser. The PPr system is housed in 8 crates with 124 modules in total. Each PPr module hosts $16 \mathrm{MCMs}$ and processes 64 channels.

The Cluster Processor (CP) applies sliding window algorithms to search for localised energy depositions from electron, photon and tau candidates. The sliding window is a combination of three different thresholds here, namely the energy sum in an area of $1 \times 2$ or $2 \times 1$ inside a $2 \times 2$ core in the electromagnetic layer, the energy sum of 12 neighbouring trigger towers around the core in the same layer for isolation requirements, and the hadronic layer to distinguish hadronic from electromagnetic showers. The CP system consists of 56 modules, housed in four crates.

The Jet-Energy-Sum Processor (JEP) applies similar algorithms as the CP to identify hadronic jets. As jets cover a larger area in $\eta-\phi$ space, the JEP operates with a coarser granularity of $0.2 \times 0.2$ and combines the electromagnetic and hadronic layer to so-called jet elements. The sliding window is available in three different sizes, namely $2 \times 2,3 \times 3$ or $4 \times 4$ jet elements, analogous to different radii for cone-like jet algorithms. The JEP also determines the total energy of the event from the scalar sum of the transverse energy in all jet elements, as well as the missing transverse energy from their vectorial sum. The JEP system comprises 32 modules, which are grouped into two crates.

The Common Merger Modules (CMM) count the number of hits above threshold in each crate of the CP and JEP systems. The combined information is transmitted to the Central Trigger Processor (CTP), which also receives signals from the Level1 muon trigger (L1Muon). The trigger decision at Level-1 is based on the multiplicity of trigger thresholds that are fulfilled in a given event. The CTP issues a Level-1 Accept (L1A) signal if the trigger multiplicity of a single or a combination of thresholds exceeds a certain value as defined in the trigger menu. For the accepted events, the Regions of Interest (RoI) are passed to the Level-2 trigger and the trigger information is read out via the Read-Out Drivers (RODs).

The total latency of the Level-1 Calorimeter Trigger is $2.1 \mu$ s, i.e. well below the length of the on-detector pipeline memories where the full event information is buffered until the Level-1 trigger reaches a decision.

\section{L1CALO OPERATION IN RUN 1}

During the first data taking phase of Run 1, L1Calo was operated very successfully. It provided full efficiency for

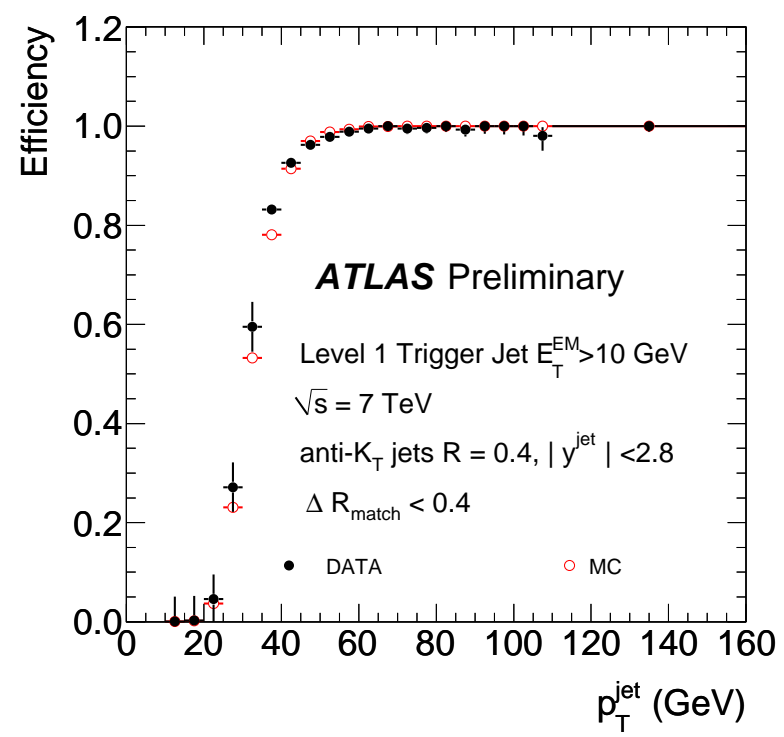

Fig. 2. Trigger efficiency for an anti- $k_{T}$ jet with $R=0.4$ to satisfy the Level1 trigger item $\mathrm{J} 10$ as a function of the jet $p_{T}$, integrating over $|y|<2.8$. [2].

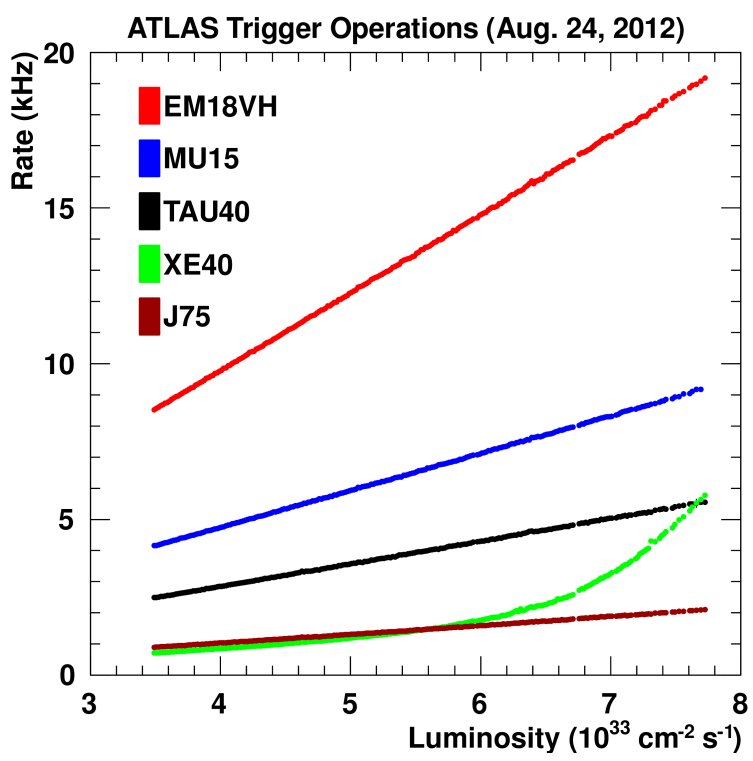

Fig. 3. Trigger rates for various trigger items as a function of the instantaneous luminosity [3].

various physics objects above certain thresholds with sharp turn on curves. Figure 2 shows the trigger efficiency for a Level-1 jet trigger J10 in a dataset corresponding to an integrated luminosity of $17 \mathrm{nb}^{-1}$. The operation was stable and robust to the change of various system parameters like noisy or dead cells. Figure 3 shows the trigger rate at Level-1 for several trigger items as a function of the instantaneous luminosity. For the single object triggers like electrons (EM18VH), taus (TAU40) and jets (J75), a linear dependency is found, indicating that the trigger items behave as expected with increasing luminosity. However, for global energy sums like missing transverse energy ( $\mathrm{XE} 40)$ as well as for low energy multijet triggers (not shown), the rates show a strong nonlinear behaviour for high instantaneous luminosity. 


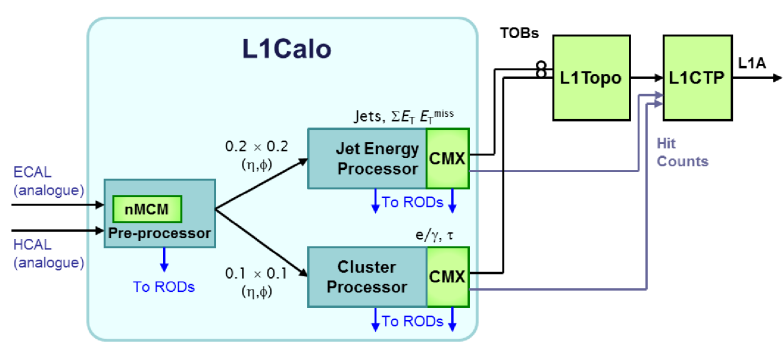

Fig. 4. Block diagram of the Level-1 Calorimeter Trigger system after the Phase 0 upgrade [4]. New components are shown in green.

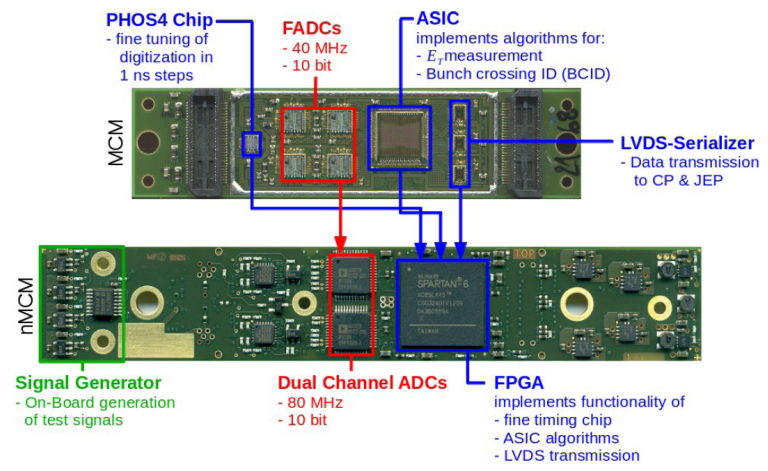

Fig. 5. Comparison of the PPr Multi-Chip Module (MCM) and the new Multi-Chip Module (nMCM)

This property was traced back to pile-up effects in signals from the LAr calorimeter. These signals are characterised by a short rise time of $50 \mathrm{~ns}$ and a long undershoot of about $450 \mathrm{~ns}$. As the complete pulse is charge neutral, the signal pedestal is constant on average. However, after longer gaps without collisions, as they are present in the filling scheme of the collider, the signals add up to a effective positive shift of the baseline. While the impact to objects which cover only a relatively small area like electrons is almost negligible, a small effect can be observed for low threshold jets. The biggest effect can be seen for total and missing energy sums, since all trigger towers contribute to these items.

\section{Phase 0 UPGRAde}

Based on the lessons learned during Run 1 and in preparation for the additional challenges to be expected with higher energy and luminosity in Run 2, a series of upgrades is planned for the L1Calo system. An overview about the upgraded system is given in the block diagram in Figure 4. During the initial upgrade three new components are currently being built:

\section{A. The new Multi Chip Module (nMCM)}

In the Phase 0 upgrade, the existing MCM on the PPr system will be replaced by the new Multi-Chip Module (nMCM). The base functionality of the $\mathrm{nMCM}$ is identical to the MCM, but the ASIC of the current system is replaced by an FPGA. As before, the $\mathrm{nMCM}$ performs the digitisation and preprocessing of the input data. Beyond this, the modern FPGA greatly improves the flexibility and allows to implement new algorithms. In particular the pile-up effects encountered during Run 1 can
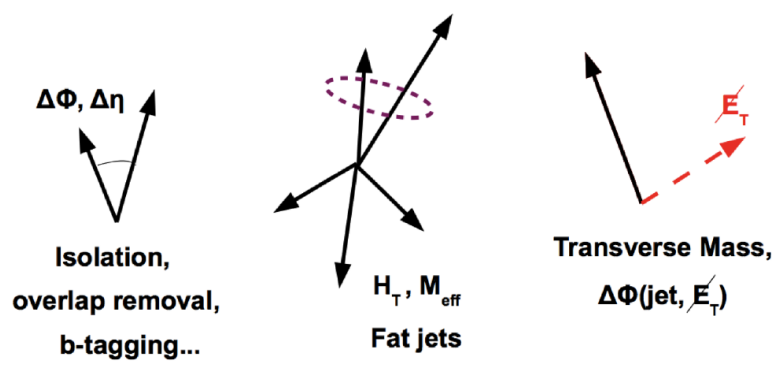

Fig. 6. Typical quantities that can be used in the Level-1 Topological Trigger.

be addressed now. The firmware of the nMCM comprises a dynamic pedestal subtraction to mitigate the out-of-time pileup effects described above. Improvements for in-time pile-up can be achieved by using an auto-correlation filter, based on the correlation between ADC samples, instead of the matched filters used currently. Furthermore, the new module allows to provide different calibrations for the CP and JEP data path with two separate look-up tables. While previously the energy calibration was done at the electromagnetic scale for both, a dedicated and optimised calibration to the hadronic energy scale can be applied. Another improvement is possible by means of the $80 \mathrm{MHz}$ ADCs on the nMCM. The better time resolution can be used to improve the so-called saturated BCID algorithm, which is used to determine the (unknown) peak position for saturated signals. The algorithms makes use of the double fequency sampling of the rising edge of the signal to extrapolate the peak position.

The nMCM incorporates the fine timing for precise sampling of the signal peak in one chip, overcoming minor shortcomings of the fine timing in its previous implementation. In addition it comprises a programmable analogue signal generator which can be used to test the full signal chain in a stand-alone setup. A visual comparison of the MCM and $\mathrm{nMCM}$ is shown in Figure 5. The production, testing and installation of $2048 \mathrm{nMCMs}$ is completed. Currently the system is commissioned and calibrated.

\section{B. The Level-1 Topological Trigger}

The Level-1 Topological Trigger (L1Topo) is a Level-1 sub-system introduced for Run 2. It gives the possibility to combine the information from electron/photon, muon, tau and jet candidates as well as the energy sums. From these objects, L1Topo calculates a variety of topological quantities such as the angular distance between different objects to apply isolation criteria, the effective mass for large- $\mathrm{R}$ jets (built from other jets), or $\Delta \phi$ between a jet and the missing energy, as illustrated in Figure 6.

The analysis of topological properties of the events greatly enhances the capabilities of the system e.g. in Higgs studies of the process $Z H \rightarrow \nu \bar{\nu} b \bar{b}$. In Run 1 , this process was selected using the missing transverse energy from the invisibe decay of the $Z$-boson into neutrinos. The L1Topo allows to select this event topology much more efficiently, requiring $\Delta \phi\left(E_{\mathrm{T}}^{\mathrm{miss}}\right.$, jet $)>1$, using the radial distance between the 


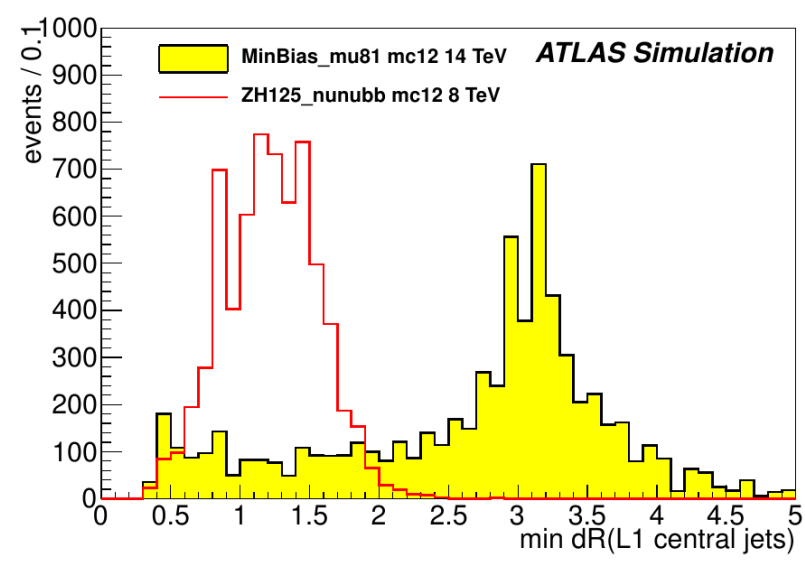

Fig. 7. Opening angle $\Delta R$ between Level-1 central jets with $p_{\mathrm{T}}<20 \mathrm{GeV}$ and $|\eta|<2.5$ in dijet events in simulation of minimum bias events (filled histogram) and $\mathrm{ZH}$ events with invisible decay of the $\mathrm{Z}$ boson (open red histogram) [4].

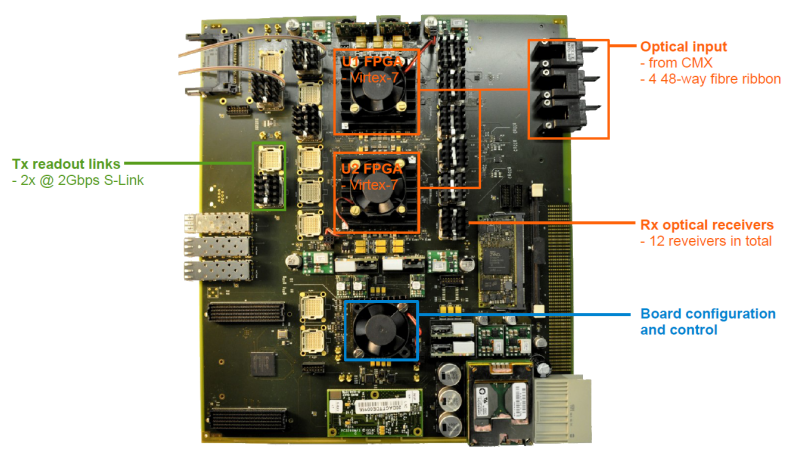

Fig. 8. Picture of the Level-1 Topological Trigger blade, with additional information as an overlay.

Level-1 central jets with $p_{\mathrm{T}}<20 \mathrm{GeV}$ and $|\eta|<2.5$, as shown in Figure 7.

L1Topo consists of two processor blades hosted in a single ATCA shelf which is located close to the CTP to minimise the signal latency. The system can be equipped with additional processor blades to accomodate more algorithms if necessary. A blade hosts two Xilinx Virtex-7 FPGAs with 80 MGTs, which operate in parallel on the same input. Each FPGA can perform different trigger algorithms independently of each other, but an additional real-time communication between the two is available as well. The input to the L1Topo are socalled trigger objects (TOBs) from the L1Calo and L1Muon systems. They are provided by the new extended CMMs (see below) and transmitted via four 48-way fibre ribbon bundles into the blade. The opto-electrical conversion is performed by 14 Avago MiniPOD optical receivers. This results in a baseline payload of about $5.1 \mathrm{~Gb} / \mathrm{s}$ for each of the 160 inputs to the two main FPGAs. The read-out of the L1Topo blades is based on two $2 \mathrm{~GB} / \mathrm{s}$ HOLA S-LINKs.

The L1Topo sub-system is currently under commissioning and the final production blades are under assembly.

\section{The Common Merger module extended (CMX)}

The Common Merger eXtended (CMX) module replaces the CMM module of the Run 1 system. It is designed to provide

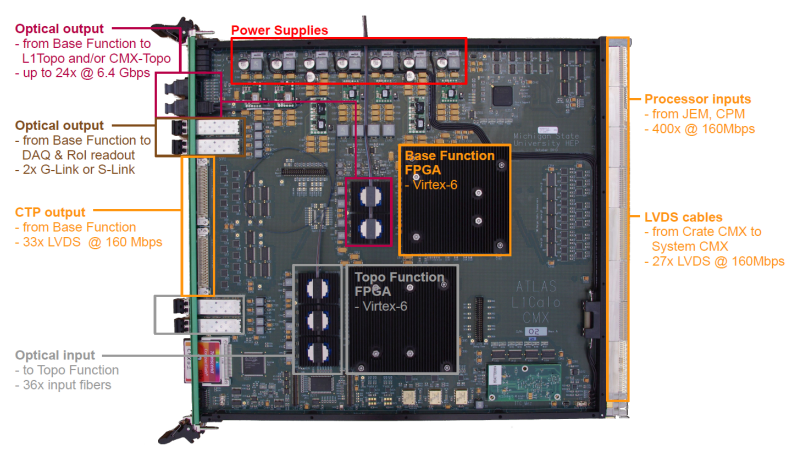

Fig. 9. Picture of the CMX module with additional information about the used hardware and data transmission as an overlay.

the same functionality as the CMM, but additionally transmits the TOBs from the various processors to the topological processor. Hence, it is a key interface which enables the operation of L1Topo.

While the CMM combined the information about trigger multiplicities from the CP and JEP systems, the CMX receives the TOBs information. The CMXs are common modules which are hosted in each of the processor crates. To cope with the larger amount of data, the transmission frequency from the processors to the CMX is increased with respect to the CMM from $40 \mathrm{Mb} / \mathrm{s}$ to $160 \mathrm{Mb} / \mathrm{s}$. The CMX is equipped with two Virtex-6 FPGAs, and the installed firmware varies depending on the . The Base-CMX FPGA is sustaining the functionality of the CMM, counting the number of TOBs over threshold and providing this multiplicity to the CTP. The processing of TOBs also includes threshold-dependent isolation criteria for the CP objects and different jet sizes for the JEP objects, which for Run 1 had been evaluated directly on the CP and JEP, respectively. A benefit from the large size of the FPGA logic is that the number of available thresholds can be increased easily for Run 2 .

In addition to the evaluation of the multiplicity information, the Base-CMX FPGA also prepared the TOB information for transmission to the L1Copo processor. This includes zero suppression and grouping of the TOBs. Two copies of the data are transmitted from the Base-CMX FPGA via twelve $6.4 \mathrm{~Gb} / \mathrm{s}$ output fibres each, hence supporting two L1Topo modules. For further upgrades of the system, CMX can be equipped with another FPGA (TP-CMX FPGA) to perform additional computations for certain TOBs.

Figure 9 shows a picture of the CMX module with additional information about the used hardware and data transmission as an overlay. The production of the CMX is completed, and the installation at CERN is almost finished.

\section{Phase 1 Upgrade}

During the Phase 1 upgrade, the read-out of the LAr based calorimeter system will be replaced by optical data transmission. In the course of this replacement, the Level-1 Calorimeter Trigger system undergoes a substantial redesign, switching to optical inputs and new processor hardware. An optical plant provides a uniform distribution of the input to three new processors: the electron feature extractor (eFEX), 


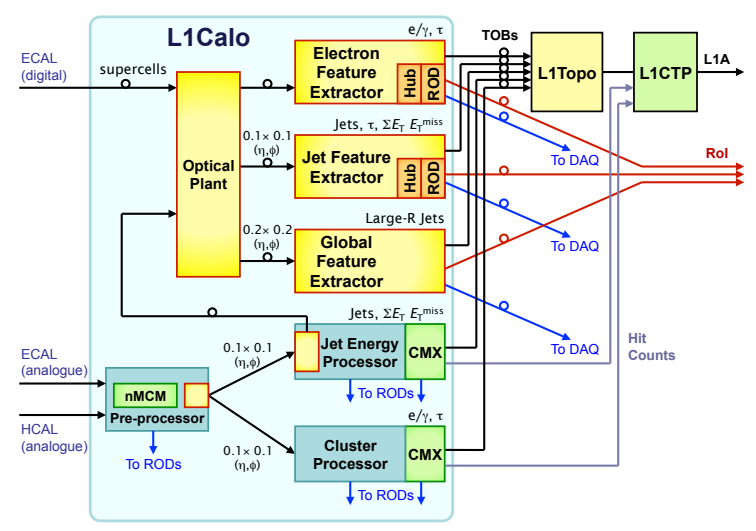

Fig. 10. Block diagram of the Level-1 Calorimeter Trigger after the Phase 1 upgrade. New components are shown in yellow/orange.

the jet feature extractor (jFEX) and the global feature extractor (gFEX). The employed techniques to select trigger candidates are mostly the same as in the previous system, e.g. sliding windows and energy sums, but at higher granularity, better resolution in a more flexible configuration. This allows e.g. to employ shower shape variables for the electron selection. In addition, the new processors also represent a major preparation for the next upgrade phase beyond Run 3.

The input to the optical plant is split into the input from the LAr based calorimeter system (ECAL) and the calorimeter system based on scintillating tiles (HCAL). For the ECAL read-out on one hand, digitisation and preprocessing of the signals will be performed on-detector, in addition to a tenfold increase of the provided granularity. These so-called supercells represent a subdivision of the $0.1 \times 0.1$ trigger tower area into 4 longitudinal segments, where the inner two layers are subdivided further into $0.25 \times 0.1$ strips in $\eta-\phi$. The HCAL read-out on the other hand remains unchanged for the Phase 1 upgrade. Hence, the analogue read-out of the existing L1Calo system is retained for those channels, but an electric-optical signal conversion is added, modifying the output and input stage of the PPr and JEP systems, respectively. From the optical plant, the input signals are distributed to the subsequent processors in three different granularities, satisfying the needs for the different object selection they aim for.

The eFEX identifies isolated energy deposits to select electron, photon and tau candidates. It receives the full information from the new supercells. The high granularity is expected to provide a significantly improvement of the electron identification in the high luminosity environment. The jFEX performs jet algorithms and calculates the energy sums as the JEP system, but also includes the search for large-area tau candidates. It uses a granularity of $0.1 \times 0.1$ in $\eta-\phi$. The gFEX is a new component for large- $\mathrm{R}$ jets with window sizes of up to $1.8 \times 1.8$ at a granularity of $0.2 \times 0.2 \mathrm{n} \eta-\phi$. The design includes an event-by-event pile-up suppression, as required for such large-area objects.

The output path of trigger multiplicities to the CTP and TOBs to L1Topo, formerly handled by the CMX, will be streamlined. In the new design, TOBs are sent to L1Topo directly from the feature extractor modules. The determination of trigger multiplicities is then performed by the L1Topo system on basis of the TOBs. The trigger data acquisition and the transmission of the RoIs to the higher trigger levels is attributed to Hubs housed in the same crate as the FEX modules.

The Phase 1 upgrade is currently in its initial design phase. A major challenge in the design are the extreme data transmission rates. The entire system will be implemented using the Advanced Telecommunications Computing Architecture (ATCA), replacing the VME bus architecture of the initial system. Higher speed links providing $10 \mathrm{~GB} / \mathrm{s}$ are currently under investigation.

The existing L1Calo system will serve as legacy system in the beginning of Run 3 until the new processors are fully commissioned.

\section{SUMMARY}

After successful operation during Run 1, the Level-1 Calorimeter Trigger faces new challenges in future running. In order to cope with these, a stepwise upgrade program has been developed, which makes use of the advancement of technology and keeps parts of the system unchanged to guarantee a working trigger by mitigation of the overall risk.

\section{REFERENCES}

[1] ALTAS Collaboration, The ATLAS Experiment at the CERN Large Hadron Collider, JINST 3 (2008) S08003.

[2] ATLAS Collaboration, Performance of the ATLAS Jet Trigger in the Early $\sqrt{s}=7 \mathrm{TeV}$ Data, ATLAS-CONF-2010-094, Geneva, Switzerland, 2013.

[3] https://twiki.cern.ch/twiki/bin/view/AtlasPublic/TriggerOperationPublicResults

[4] R. Bartoldus et al, Technical Design Report for the Phase-I Upgrade of the ATLAS TDAQ System, CERN-LHCC-2013-018, ATLAS-TDR-023. Geneva, Switzerland, 2013. 